\title{
Computational Modelling of Cardiac Trabecula Mechanics
}

\begin{abstract}
A Schroeder $^{1} \quad$ TP Babarenda Gamage ${ }^{2} \quad$ V Wang ${ }^{3}$ DS Loiselle ${ }^{4} \quad$ PMF Nielsen $^{5} \quad$ DP Nickerson ${ }^{6}$ $\begin{array}{llll}\text { M Cheuk } & \text { AJ Taberner } & & \text { MP Nash }\end{array}{ }^{9} \quad \mathrm{~K} \operatorname{Tran}^{10}$
\end{abstract}

Recieved 26 November 2017; Accepted 12 January 2018

\begin{abstract}
Cardiac trabeculae are thin strips of muscle within the ventricles that can be readily excised and used to investigate contractile mechanics of cardiac muscle. Recently, the Auckland Bioengineering Institute has developed a novel cardiac myometer that simultaneously measures force, length and shape of actively contracting isolated cardiac trabeculae. Here we have developed a muscle-specific computational model based on optical coherence tomography geometric surface data that replicates passive mechanics of trabecula using a Guccione constitutive relation. We hypothesised that the muscle's surface geometry data, in addition to force-length data, would improve the fit between the model simulated mechanics and the experimental data. The trabecula
\end{abstract}

DOI:10.21914/anziamj.v59i0.12682, (C) Austral. Mathematical Soc. 2018. Published April 24, 2018, as part of the Proceedings of the 13th Biennial Engineering Mathematics and Applications Conference. ISSN 1445-8810. (Print two pages per sheet of paper.) Copies of this article must not be made otherwise available on the internet; instead link directly to the DOI for this article. 
model was optimised using two different objective functions (muscle length or shape) driven by a pressure boundary condition. For both objective functions, there was a region of optimal parameters the optimiser tended towards but, due to the coupling between parameters, the ability to find the true optimal parameters was hindered. Due to the limitations of the data, we found that the addition of surface data did not improve parameter estimation and that using only the force-length data provided sufficient information to produce an optimal fit.

\section{Contents}

1 Introduction

2 Methods

2.1 Data Acquisition . . . . . . . . . . . . . . . C32

2.2 The Model . . . . . . . . . . . . . . . . . . . . . . . C C32

2.3 The Constitutive Equation . . . . . . . . . . . . C34

2.4 Objective Functions . . . . . . . . . . . . . C C34

2.5 Model Verification ............... . . C35

3 Results

C36

4 Discussion

4.1 Combining Objective Functions . . . . . . . . . . C42

4.2 Parameter Coupling . . . . . . . . . . . . C44

5 Conclusions

C45

References

C45 


\section{Introduction}

A popular approach for investigating the mechanisms behind cardiac muscle mechanics is the use of computational models. In this study, we developed a computational model of passive trabecula mechanics which we used to optimise the parameters of a modified Guccione, McCulloch, and Waldman [6] constitutive equation. The experimental data for our study were acquired from a preparation of rat cardiac trabecula extracted from the right ventricle of the heart. Cardiac trabeculae are the smallest naturally arising collections of linearly arranged heart muscle cells and are the most widely used experimental muscle preparations for studying heart muscle. The linear nature of trabeculae enables researchers to model stresses and strains along the length of muscle cells.

The Auckland Bioengineering Institute (ABI) has previously developed a novel cardiac myometer which is capable of measuring muscle force and displacement (among other measurements) of a trabecula undergoing fixed-length contractions [5]. More recently, an optical coherence tomography (OCT) system was incorporated into the cardiac myometer for measuring the geometric shape changes of cardiac trabeculae in vitro during a contraction [4]. The cardiac myometer measures the muscle displacements but the regional stresses and muscle constitutive properties cannot be measured experimentally, thus, computational models are required to interpret the experimental observations. We have developed a muscle-specific computational model from OCT geometric surface data that replicates passive trabecula mechanics. We hypothesised that the use of trabecula surface geometry during parameter estimation decrease the difference between the model predicted measurements and the experimentally observed measurements. To the best of the authors' knowledge, this is the first study in which experimentally obtained muscle surface data were used to develop a computational model of cardiac trabecula mechanics. 


\section{Methods}

\subsection{Data Acquisition}

The experimental data were collected from a single trabecula extracted from the right ventricle of a rat. The trabecula was mounted in the cardiac myometer via two platinum hooks which can be controlled for muscle length and position [1]. The mounted trabecula was placed in a capillary of flowing nutrients and oxygen for the remainder of the experiment [7]. The trabecula was stretched to five different lengths and electrically stimulated at a frequency of $2 \mathrm{~Hz}$. The reference muscle length was $2.01 \mathrm{~mm}$ ( $75 \%$ of the maximum length). From the reference length, the muscle was stretched to five different lengths: $2.12 \mathrm{~mm}$ (80\%), $2.24 \mathrm{~mm}$ (85\%), $2.35 \mathrm{~mm}$ (90\%), $2.46 \mathrm{~mm}$ (95\%), and $2.57 \mathrm{~mm}(100 \%)$. To measure the force produced by the trabecula at each length, the downstream hook was attached to a thin stainless steel beam whose deflection was tracked via laser diffraction. To gather the passive force we averaged the diastolic force at each trabecula length to provide the corresponding passive force-length relationship (Figure 1). The reference length was taken to be stress-free and the passive forces were offset to reflect this. The surface geometry of the trabecula was measured using OCT mounted with a full view of the trabecula.

\subsection{The Model}

Finite element models have been used extensively for investigating the mechanics of the whole heart, but not for cardiac trabecula. Here, we developed a finite element model of rat cardiac trabecula to investigate it's passive mechanics. The geometric mesh was composed of eight cubic Lagrange 16-node elements. The mechanical behaviour of the muscle was modelled using the Guccione, McCulloch, and Waldman [6] constitutive equation (Section 2.3). The mesh was fitted to the geometric data for the reference muscle length, 


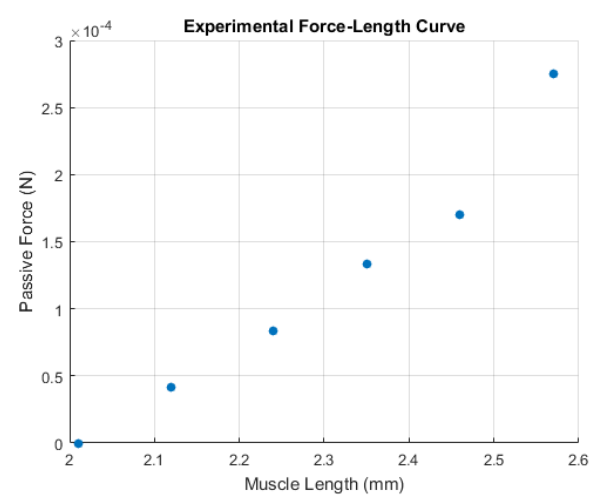

Figure 1: Experimentally measured passive force-length relationship of a cardiac trabecula. This dataset was used to parameterise the passive properties of our computational trabecula model.

which became the reference state of the model. Initially, the OCT data's axis of stretching did not align with a coordinate direction in the model, so we transformed the dataset to align the length of the muscle with the z-axis of the model's coordinate system. To transform the data we performed a rigid transformation using the OCT data by minimising the root mean square error (RMSE) between the OCT data coordinates for all extension lengths and the model's z-axis.

To ensure the model's simulated passive stretching matched with the experimental observations, the constitutive properties of the trabecula were estimated using optimisation. The model was fitted to the experimental data by optimising the parameters of the Guccione, McCulloch, and Waldman [6] equation using OpenCMISS [3]. The parameter set output from the optimising routine were the parameters that gave the best match between the model prediction and the data provided and represented the mechanical properties of our trabecula. 


\subsection{The Constitutive Equation}

The equation governing the constitutive behaviour of the models are a modified version of the Guccione, McCulloch, and Waldman [6] equations for threedimensional transverse-isotropic cardiac tissue as described by Nash and Hunter [8]. The strain energy function, $\bar{W}$ is defined as

$$
\bar{W}=\frac{C_{1}}{2}\left(e^{Q}-1\right),
$$

where

$$
Q=C_{2} E_{f f}^{2}+C_{3}\left[E_{s s}^{2}+E_{n n}^{2}+2\left(E_{s n} E_{n s}\right)\right]+2 C_{4}\left[E_{f n} E_{n f}+E_{f s} E_{s f}\right] .
$$

$\mathrm{Q}$ is a quadratic function of the three strain components defined with respect to the micro-structural coordinates. E refers to components of the Green's strain tensor with $E_{f f}$ representing strain in the fibre direction, and $E_{s s}$ and $E_{n n}$ representing strain in the two transverse directions. All other terms in Green's strain tensor represent shear. $\mathrm{C}_{1}$ is a universal scaling coefficient, $\mathrm{C}_{2}$ and $\mathrm{C}_{3}$ scale the nonlinearity of the stress-strain relation in the fibre and transverse directions, respectively, and $\mathrm{C}_{4}$ scales the shear components. The four parameters of the Guccione constitutive equations $\left(\mathrm{C}_{1}\right.$ to $\left.\mathrm{C}_{4}\right)$ are empirical and give no direct interpretation of tissue properties.

\subsection{Objective Functions}

Using a least-squares optimiser, we explored the use of two different objective functions to get an estimate of the constitutive properties of the trabecula, which cannot be experimentally measured. Our Displacement Model's objective function minimised the difference between the predicted and observed muscle lengths while that for the Projection Model minimised the difference between the predicted and observed muscle shape. The general form of the error measure (f) provided to the least-squares optimiser was:

$$
f=\left[X_{1}, X_{2}, X_{3}, X_{4}, X_{5}\right] \text {, }
$$


where

$$
X_{m}=P_{m}-E_{m} .
$$

$X_{m}$ is the error per length step $m, P_{m}$ is the model prediction for length step $m$ (muscle length or shape), and $E_{m}$ is the experimental observation for length step $m$. For the Displacement Model, $\mathrm{P}_{m}$ and $\mathrm{E}_{\mathrm{m}}$ represent muscle lengths. For the Projection Model, they represent the geometric model surface and the experimental OCT data, respectively. To characterise the model fits we calculated the RMSE of $f$ for the parameter sets obtained via the optimisation of each objective function.

\subsection{Model Verification}

Before applying the objective functions to a model to match experimental data, we first tested them on a model generated from synthetic data. Material parameters were assigned to the synthetic model and then the model was stretched to different, specified lengths in order to gather the nodal forces at the end nodes for each length step. Using the synthetic length and force data we confirmed that both of our objective functions found the assigned material parameters via optimisation.

In addition to calculating the RMSE of our models we also conducted 4D parameter sweeps and analysed the stress-strain properties of the trabecula models. Of the four parameters in the modified Guccione constitutive equation, $\mathrm{C}_{4}$ was independent of all the other parameters because the model underwent uniaxial extension and no shear components were involved. A 4D-plot of the RMSE as functions of the three identifiable parameters $\left(C_{1}, C_{2}\right.$, and $\left.C_{3}\right)$ was constructed for each objective function to identify parameter-couplings. It is known that there is coupling between parameters in the Guccione constitutive equations due to its nonlinear properties. After finding a set of parameters via optimisation, we examined the corresponding mechanical behaviour of the trabecula by looking at the relationship between stress (Cauchy-stress 
tensor) and strain (Green-Lagrange strain tensor) in the fibre and transverse directions.

\section{Results}

By varying the parameters that characterised the trabecula's passive mechanical properties, we found a region of parameter sets that minimised the fitting error between the model and the experimental data via a gradient descent method. We hypothesised that a model optimised to fit surface geometry would be more accurate compared to a model optimised to fit muscle length. However, using the surface projection errors to drive the calculation of the objective function resulted in a large discrepancy between the Projection Model's predicted muscle length and the observed muscle length. To characterise the model fit, we examined each model's muscle length error and projection error between the OCT data and the model. For two sets of arbitrary parameters with similar RMSE's, the Projection Model over-predicted muscle length by $304 \mu \mathrm{m}$ for one set of parameters and under-predicted muscle length by $216 \mu \mathrm{m}$ in the other set (Table 1). To improve the model fit, we modified the objective function of the Projection Model to also penalise differences in muscle length. The thinking behind combining the optimisation of muscle length and muscle geometry was that every direction would be constrained by experimental data and would improve the model's fit with the data. This model was labeled the Combined Model.

For each objective function, there was an optimal combination of parameters that would give rise to the smallest error between the model and the experimental data. The optimal parameters for each model reside in a range of plausible parameter combinations known as the parameter indifference region. The Projection Model possessed a large parameter indifference region because many different combinations of parameters gave rise to similar RMSE's. For example, a change in the mean projection error from $8.64 \pm 9.30 \mu \mathrm{m}$ to $9.15 \pm 9.42 \mu \mathrm{m}$ and a change in the mean muscle length error from 
$199 \pm 61.5 \mu \mathrm{m}$ to $182 \pm 68.6 \mu \mathrm{m}$ only caused a small change in the RMSE of the Projection Model, as seen in Parameter Sets 1 and 2 of Table 1. This attributed to a large parameter indifference region in which many combinations of parameters would give rise to a similar error between the model and the experiment.

Conversely, for comparatively smaller changes in muscle length error and projection error the Displacement Model saw changes in the RMSE that were of similar magnitude to those of the Projection Model (Table 1, Parameter Sets 3 and 4). This means the Displacement Model was more sensitive to changes, and this attributed to a more defined parameter indifference region. When we combined the Displacement and Projection Model's objective functions to create the Combined Model we found that the Projection Model's insensitivity to changes in parameters resulted in a less defined parameter indifference region for the Combined Model when compared to the Displacement Model. This can be seen by the dark green valleys in Figure 2 which represent portions of each model's parameter indifference region and correspond to parameter sets that gave rise to the lowest RMSE. The contour plots for the Combined Model show a more gradual descent when compared to the contour plots for the Displacement Model, indicating a wider range of plausible parameter sets that produce very similar stress-strain behaviours. Implementing the Combined Model decreased the identifiability of the constitutive parameters and also did not improve the accuracy of fitting the model to the experimental data (Table 3). It is also possible that the insensitivity in the optimisation of surface geometry data was because the mode of deformation performed in the experiment was uniaxial. Since the trabecula was only deformed in the fibre direction the force-length relationship may be sufficient information to replicate the experiment. Both the Displacement and the Combined Models produced stress-strain curves in the fibre and transverse directions of the trabecula under uniaxial loading conditions with the same accuracy (Figure 3).

In order to compare our models, the optimisation for each objective function was initialised with the same randomly generated parameters $(0.024,1.46$, 1.16). The resulting optimal parameters for each model are summarised in 
Table 1: The root mean square error (RMSE), length error, and projection error for two parameter sets chosen from the Projection Model's parameter indifference region (Parameter Sets 1 and 2), and two parameter sets chosen from the Displacement Model's parameter indifference region (Parameter Sets 3 and 4). The parameter sets correspond to $\left(\mathrm{C}_{1}, \mathrm{C}_{2}\right.$, and $\left.\mathrm{C}_{3}\right)$. The maximum projection error was $60.1 \mu \mathrm{m}$ for all four simulations.

\begin{tabular}{lcccc}
\hline Parameter Set & $\begin{array}{c}\text { RMSE } \\
(\mu \mathrm{m})\end{array}$ & $\begin{array}{c}\text { Muscle Length } \\
\text { Error } \\
(\mu \mathrm{m})\end{array}$ & $\begin{array}{c}\text { Max Muscle } \\
\text { Length } \\
\text { Error }(\mu \mathrm{m})\end{array}$ & $\begin{array}{c}\text { Projection } \\
\text { Error } \\
(\mu \mathrm{m})\end{array}$ \\
\hline $1(0.022,1.5,1.3)$ & 12.69 & $-119 \pm 61.5$ & -216 & $8.64 \pm 9.30$ \\
$2(0.017,1.1,1.0)$ & 13.13 & $182 \pm 68.6$ & 304 & $9.15 \pm 9.42$ \\
$3(0.024,1.1,0.9)$ & 39.09 & $-1.10 \pm 39.1$ & -71.1 & $8.65 \pm 9.24$ \\
$4(0.020,1.3,1.1)$ & 37.21 & $-0.50 \pm 37.2$ & -70.0 & $8.59 \pm 9.24$ \\
\hline
\end{tabular}

Table 2. There is no set of parameters reported for the Projection Model because the optimiser was too insensitive to changes in the projection errors and would continually stretch the muscle until it reached a set of parameters that did not converge. As stated in the Methods section, $\mathrm{C}_{4}$ was independent of the other three parameters because of the absence of shearing. In all of our models we fixed $\mathrm{C}_{4}$ to a constant value of 1.083 . Initial optimisations showed that drastic changes in the value of $\mathrm{C}_{4}$ had very little effect on the model, therefore, the value $\mathrm{C}_{4}$ was of little consequence and chosen arbitrarily. The parameters that we found via optimisation are consistent with Wang et al. [10], Augenstein et al. [2], and Omens, MacKenna, and McCulloch [9] in that the stiffness in the fibre direction was higher than in the transverse directions. When comparing the parameters obtained in this study to the parameters obtained in previous studies (Table 4) our parameters are significantly smaller. The differences are not well understood, but possible reasons for the differences are the variations in species and test types utilised by each study. Wang et al. [10] used in vivo magnetic resonance imaging on a dog heart, Augenstein et al. [2] used an isolated, arrested porcine heart, and Omens, MacKenna, and McCulloch [9] used an isolated, arrested rat heart. 
Table 2: Optimal parameter sets obtained via optimisation of the Displacement Model and the Combined Model along with their corresponding root mean square error (RMSE).

\begin{tabular}{lcccc}
\hline Objective Function & $\begin{array}{c}\mathrm{C}_{1} \\
(\mathbf{k P a})\end{array}$ & $\mathrm{C}_{2}()$ & $\mathrm{C}_{3}()$ & $\begin{array}{c}\text { RMSE } \\
(\mu \mathrm{m})\end{array}$ \\
\hline Displacement Model & 0.020 & 1.30 & 0.93 & 37.01 \\
Combined Model & 0.021 & 1.27 & 0.90 & 26.82 \\
\hline
\end{tabular}

Table 3: The root mean square error (RMSE), length error, and projection error for the parameter sets obtained via optimisation of each objective function (Table 2). The Projection Model is not presented because the optimiser was unable to identify a set of optimal parameters.

\begin{tabular}{lcccc}
\hline $\begin{array}{c}\text { Objective } \\
\text { Function }\end{array}$ & $\begin{array}{c}\text { RMSE } \\
(\mu \mathrm{m})\end{array}$ & $\begin{array}{c}\text { Muscle Length } \\
\text { Error }(\mu \mathrm{m})\end{array}$ & $\begin{array}{c}\text { Max Muscle } \\
\text { Length Error } \\
(\mu \mathrm{m})\end{array}$ & $\begin{array}{c}\text { Projection } \\
\text { Error }(\mu \mathrm{m})\end{array}$ \\
\hline Displacement Model & 37.01 & $2.1 \pm 36.9$ & 38.9 & $8.6 \pm 9.3$ \\
Combined Model & 26.82 & $1.5 \pm 37.2$ & 39.1 & $8.6 \pm 9.3$ \\
\hline
\end{tabular}

Table 4: Comparing the parameter set obtained using the Combined Model to previously published parameter sets.

\begin{tabular}{lcccc}
\hline \multicolumn{1}{c}{ Study } & $\mathrm{C}_{1}(\mathrm{kPa})$ & $\mathrm{C}_{2}()$ & $\mathrm{C}_{3}()$ & $\mathrm{C}_{4}()$ \\
\hline Current Study (Combined Model) & 0.016 & 1.63 & 1.23 & 1.08 \\
Wang et al. [10] & 0.831 & 14.3 & 4.49 & 0.762 \\
Augenstein et al. [2] & 3.0 & 11.1 & 1.76 & 10.0 \\
Omens, MacKenna, and McCulloch [9] & 1.1 & 9.2 & 2.0 & 3.7 \\
\hline
\end{tabular}



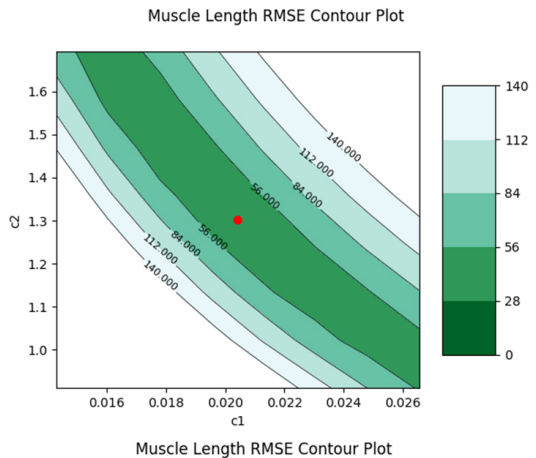

Combined Muscle Length and Projection RMSE Contour Plot

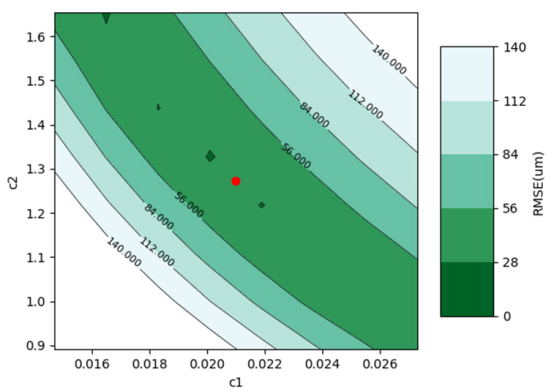

Combined Muscle Length and Projection RMSE Contour Plot
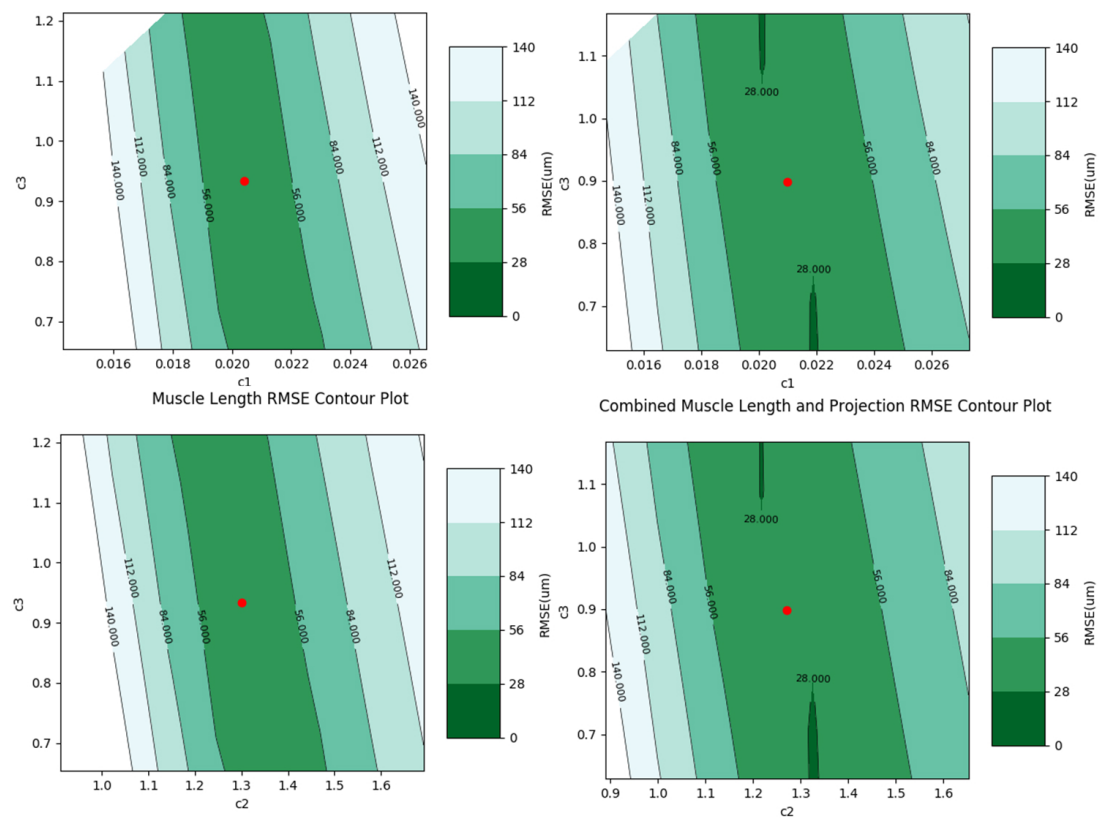

Combined Muscle Length and Projection RMSE Contour Plot

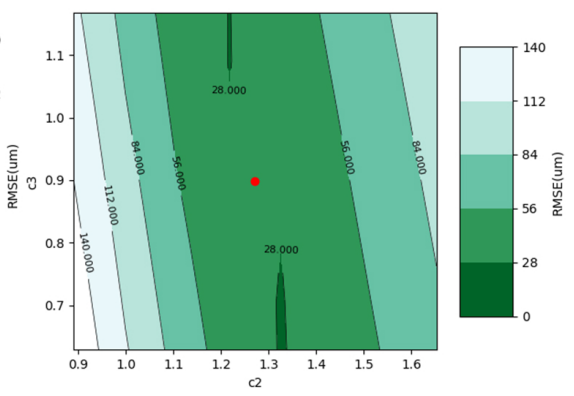

Figure 2: The three planes containing the parameters obtained via optimisation of the Displacement Model's (left) and the Combined Model's (right) objective functions. The relationships between $C_{1}$ and $C_{2}$ (top), $C_{1}$ and $C_{3}$ (middle), and $C_{2}$ and $\mathrm{C}_{3}$ (bottom) are shown. The darkest contours seen in the combined model's plots are interpolation artifacts due to the limited set of discrete parameters evaluated when generating this plot. 

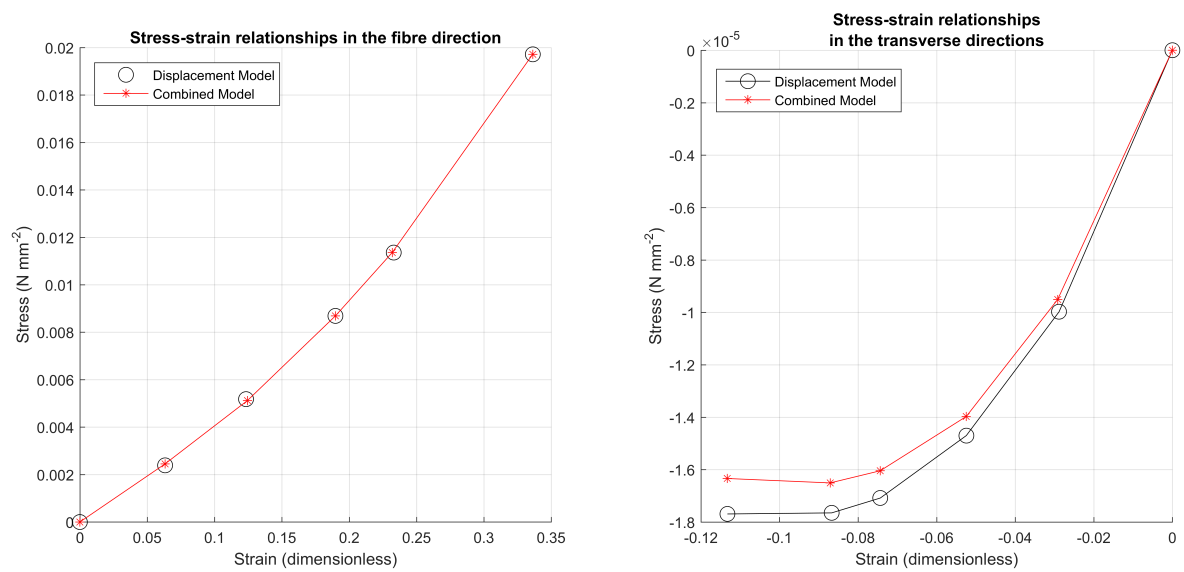

Figure 3: Stress-strain curves in the fibre (left) and transverse (right) directions simulated using the optimal parameters from the Displacement Model and the Combined Model.

\section{Discussion}

The goal of this study was to determine if adding muscle shape information would improve the fit of a model of passive trabecula mechanics. In this study, we fit one parameter to the fibre direction and one parameter to the transverse directions $\left(\mathrm{C}_{2}\right.$ and $\mathrm{C}_{3}$ respectively). If there is no inherent coupling in the constitutive relation and the stiffness of the trabecula is homogeneous along the length of the muscle, then there is one set of optimal parameters. This was confirmed with the synthetic models, which found the same set of optimal parameters regardless of the objective function that was employed and the initial starting point. Unlike the synthetic models, the models created from the experimental data did not find the same set of optimal parameters as each other, as seen in Table 2. We attributed this to the high degree of coupling between the parameters $C_{1}$ and $C_{2}$ and the mostly independent nature of $\mathrm{C}_{3}$, seen in the contour plots in Figure 2. For comparing the Displacement and the Combined Models, we looked at the length error and the projection 
error instead of the RMSE because the RMSE is only useful for comparisons within the same model, not between models. This was because the RMSE of the Displacement Model was calculated using a vector of five numbers, the length error per length step, while the RMSE of the Combined Model was calculated using a vector of ten numbers, the length and projection errors per length step. The presence of the projection errors in the Combined Model's objective function caused the RMSE of the Combined Model to be smaller than the RMSE of the Displacement Model. This made it appear as though the Combined Model had a lower error when in reality the two models behaved similarly.

\subsection{Combining Objective Functions}

When using the Projection Model there was a high degree of insensitivity to muscle length which we tried to constrain by penalising muscle length error in the combined model. The discrepancy seen between the synthetic model, which accurately matched the muscle length, and the model created from experimental data could be attributed to several factors. The synthetic data assumed we had an unloaded stress free state as the mechanics reference model. For this study, we used $75 \%$ of the longest length step as the reference state. In reality, this was not completely stress free but we had to make that assumption given that it was the lowest length step measured in the experiment. Another factor that could have contributed to the observed discrepancy in muscle length was our assumption that we could measure the end locations and displacements of the trabecula accurately. Past data gathered on the cardiac myometer have shown that some trabeculae have slippage around the mounting hooks causing parts of the muscles to come into the viewing plane in the longest length steps that weren't present in the undeformed state. This contributes to an inaccurate measurement of muscle displacements and end locations, and could be a reason why our Projection Model consistently had large muscle length errors. 

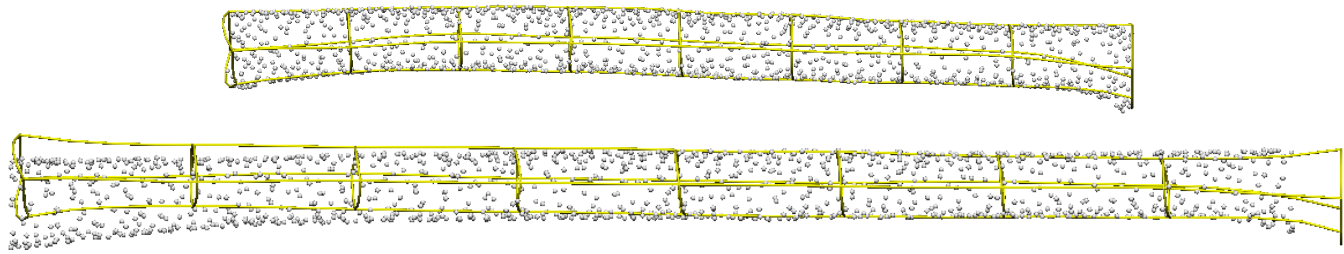

Figure 4: The alignment between the model and the surface geometry data cloud in the undeformed state (top) and the longest length step (bottom).

When running the Projection Model, the optimiser was less sensitive to the stiffness in the fibre direction and had a greater sensitivity to the stiffness in the transverse directions. This is contrary to the Displacement Model, which was more sensitive in the fibre direction than in the transverse directions. This was expected as the Projection Model was optimised to fit data in the transverse directions, while the Displacement Model was optimised to fit data in the fibre direction. The decreased sensitivity to changes in parameters seen in the Projection Model can also be due to the discrepancy between the model's axis of extension and the experimental axis of extension. When stretched to the longest length step there was a misalignment between the two axes. Figure 4 shows the alignment of the data compared to the model for the reference state and the longest length step and clearly shows the data point cloud rotating out of alignment at the left end as the muscle stretches. This can most likely be attributed to the rigid alignment of the data with the axial direction. Since the OCT data on the muscle surface are not rigid landmarks, using the data as a means to find the axis of extension led to an inaccurate alignment. While the motion of the hooks can be accurately controlled, it is possible that the muscle was not taut between the mounting hooks in the reference state and when stretched the muscle's position changed. To address this alignment problem, it is required to track the motion of the muscle, the hooks, and the linear actuator at each length.

Combining the Projection Model and the Displacement Model to create the Combined Model did not increase the ability of our model finding the reference 
material parameters. In fact, the optimal parameters of the Combined Model were less identifiable those of the Displacement Model. However, for the initial parameter set optimised in this study, the Combined Model and the Displacement Model terminated optimisation around the same region of parameters and had similar model fits. Between the two model's optimised results there was only a $4.8 \%$ difference in $\mathrm{C}_{1}$, a $3.2 \%$ difference in $\mathrm{C}_{2}$, and a $2.3 \%$ difference in $\mathrm{C}_{3}$.

\subsection{Parameter Coupling}

Though we did not find one single set of optimal parameters via optimisation, our models did find a region of possible parameter sets that gave rise to similar muscle behaviour. The similarities seen in the stress-strain curves produced by the two models, despite the different parameters sets evaluated, is a characteristic of the coupling between parameters. It is known that there is a coupling between parameters in the Guccione constitutive equations and this can clearly be seen in Figure 2. For all of our models, there was a strong coupling between $C_{1}$ and $C_{2}$, which was represented by the dark green valley that spans diagonally from one corner to the other. For a given $C_{3}$ parameter, there were multiple sets of $C_{1}$ and $C_{2}$ combinations that gave rise to a similar RMSE. Also seen in Figure 2 is the inability of our models to accurately identify $C_{3}$. This is represented in the contour plots between $C_{1}$ and $C_{3}$ as well as between $C_{2}$ and $C_{3}$ by the mostly vertical dark green valley. It is important to note that the parameter not being compared in a given contour plot was held constant at the optimal value for all parameter combinations shown in the plot. 


\section{Conclusions}

Identifying constitutive parameters of passive trabecula mechanics using OCT data, as opposed to only force-length data, did not more reliably identify the constitutive parameters. Also, the use of OCT data in combination with force/pressure data did not reduce the parameter indifference region. The coupling between parameters $\mathrm{C}_{1}$ and $\mathrm{C}_{2}$, along with the high degree of independence of $\mathrm{C}_{3}$, made identifying one set of optimal parameters for this muscle-specific passive trabecula mechanics model unattainable. However, the mechanical behaviour observed in the stress-strain plots was similar for both models. Until experiments can be conducted in which the axis of muscle extension is meticulously tracked or maintained there is no added benefit of constraining computational models using geometric surface data.

The next step is to create a simplified model of trabecula contraction that can be combined with the passive model. This could be accomplished by combining simplified versions of electrical activation, calcium dynamics, and cross-bridge cycling models. In the future, more complex models of contractile mechanics could explore the effects of heterogeneous activation and heterogeneous muscle stiffness, which may more closely resemble in vivo conditions.

Acknowledgements We would like to extend our sincerest gratitude to the Whitaker International Program, run through the Institute of International Education (IIE), for financially supporting Alison Schroeder throughout her Master of Engineering degree at the ABI.

\section{References}

[1] A. Anderson. The Cardiac Myometer: Measuring Matters of the Heart. PhD thesis, University of Auckland, 2016. C32 
[2] K. F. Augenstein, Brett R. Cowan, Ian J. LeGrice, Poul M. F. Nielsen, and Alistair A. Young. Method and apparatus for soft tissue material parameter estimation using tissue tagged Magnetic Resonance Imaging. Journal of Biomechanical Engineering, 127(1):148-157, February 2005. C38, C39

[3] C. Bradley, Andy Bowery, Randall Britten, Vincent Budelmann, Oscar Camara, Richard Christie, Andrew Cookson, Alejandro F. Frangi, Thiranja Babarenda Gamage, Thomas Heidlauf, Sebastian Krittian, David Ladd, Caton Little, Kumar Mithraratne, Martyn Nash, David Nickerson, Poul Nielsen, Oyvind Nordbo, Stig Omholt, Ali Pashaei, David Paterson, Vijayaraghavan Rajagopal, Adam Reeve, Oliver Rohrle, Soroush Safaei, Rafael Sebastian, Martin Steghofer, Tim Wu, Ting Yu, Heye Zhang, and Peter Hunter. OpenCMISS: A multi-physics \& multi-scale computational infrastructure for the VPH/Physiome project. Progress in Biophysics and Molecular Biology, 107(1):32-47, October 2011. doi:http://dx.doi.org/10.1016/j.pbiomolbio.2011.06.015 C33

[4] M. L. Cheuk, A. J. Anderson, J. C. Han, N. Lippok, F. Vanholsbeeck, B. P. Ruddy, D. S. Loiselle, P. M. F. Nielsen, and A. J. Taberner. Four-Dimensional Imaging of Cardiac Trabeculae Contracting In Vitro Using Gated OCT. IEEE Transactions on Biomedical Engineering, 64(1):218-224, January 2017. doi:http://dx.doi.org/10.1109/TBME.2016.2553154 C31

[5] M. L. Cheuk, N. Lippok, A. W. Dixon, B. P. Ruddy, F. Vanholsbeeck, P. M. F. Nielsen, and A. J. Taberner. Optical coherence tomography imaging of cardiac trabeculae. In 2014 36th Annual International Conference of the IEEE Engineering in Medicine and Biology Society, pages 182-185, August 2014. doi:http://dx.doi.org/10.1109/EMBC.2014.6943559 C31

[6] J. M Guccione, Andrew D McCulloch, and LK Waldman. Passive material properties of intact ventricular myocardium determined from a 
cylindrical model. J Biomech Eng, 113(1):42-55, 1991. C31, C32, C33, C34

[7] J. C. Han, Andrew J. Taberner, Robert S. Kirton, Poul M. Nielsen, Nicholas P. Smith, and Denis S. Loiselle. A unique micromechanocalorimeter for simultaneous measurement of heat rate and force production of cardiac trabeculae carneae. Journal of Applied Physiology, 107(3):946-951, September 2009.

doi:http://dx.doi.org/10.1152/japplphysiol.00549.2009 C32

[8] M. P. Nash and P. J. Hunter. Regional mechanics of the beating heart. In Cardiac Perfusion and Pumping Engineering, volume Volume 1 of Clinically-Oriented Biomedical Engineering, pages 83-127. WORLD SCIENTIFIC, July 2007. doi:http://dx.doi.org/10.1142/9789812775597_0004 C34

[9] J. H. Omens, D. A. MacKenna, and A. D. McCulloch. Measurement of strain and analysis of stress in resting rat left ventricular myocardium. Journal of Biomechanics, 26(6):665-676, June 1993. doi:http://dx.doi.org/10.1016/0021-9290(93)90030-I C38, C39

[10] V. Y. Wang, H. I. Lam, Daniel B. Ennis, Brett R. Cowan, Alistair A. Young, and Martyn P. Nash. Modelling passive diastolic mechanics with quantitative MRI of cardiac structure and function. Medical Image Analysis, 13(5):773-784, October 2009. doi:http://dx.doi.org/10.1016/j.media.2009.07.006 C38, C39

\section{Author addresses}

1. A Schroeder, Auckland Bioengineering Institute, University of Auckland, New Zealand.

2. TP Babarenda Gamage, Auckland Bioengineering Institute, University of Auckland, New Zealand. 
3. V Wang, Auckland Bioengineering Institute, University of Auckland, New Zealand.

4. DS Loiselle, Auckland Bioengineering Institute and Department of Physiology, University of Auckland, New Zealand.

5. PMF Nielsen, Auckland Bioengineering Institute and Department of Engineering Science, University of Auckland, New Zealand.

6. DP Nickerson, Auckland Bioengineering Institute, University of Auckland, New Zealand.

7. M Cheuk, Auckland Bioengineering Institute, University of Auckland, New Zealand.

8. AJ Taberner, Auckland Bioengineering Institute and Department of Engineering Science, University of Auckland, New Zealand.

9. MP Nash, Auckland Bioengineering Institute and Department of Engineering Science, University of Auckland, New Zealand.

10. K Tran, Auckland Bioengineering Institute, University of Auckland, New Zealand. 